Research Article

\title{
Changes in Urinary Microalbumin Levels after Correction of Hyperuricemia in Patients with Gout: An Observational Cohort Study
}

\author{
Binit Vaidya $\mathbb{D}$, Kalpana Pudasaini $\mathbb{D}$, and Shweta Nakarmi \\ National Center for Rheumatic Diseases (NCRD), Kathmandu, Nepal \\ Correspondence should be addressed to Binit Vaidya; drbinitvaidya@yahoo.com
}

Received 5 January 2020; Revised 23 February 2020; Accepted 3 March 2020; Published 1 April 2020

Academic Editor: Bruce M. Rothschild

Copyright (C) 2020 Binit Vaidya et al. This is an open access article distributed under the Creative Commons Attribution License, which permits unrestricted use, distribution, and reproduction in any medium, provided the original work is properly cited.

\begin{abstract}
Background. Gout is commonly associated with metabolic syndrome. Strong association between the serum uric acid level and microalbuminuria has also been observed in various studies. Aim. To observe the change in urinary microalbumin after uratelowering treatment in patients with gout and microalbuminuria. Methodology. A prospective, observational study was conducted at a tertiary-level rheumatic center (NCRD) in Kathmandu, Nepal. Adults diagnosed with gout using the 2015 ACR/EULAR criteria and microalbuminuria were enrolled in the study after obtaining informed consent. Sociodemographic profile and clinical history were recorded at baseline. Serum uric acid levels, spot urinary microalbumin (MAU) excretion, blood sugar, lipid profile, and blood pressure were measured at baseline, 3-month follow-up, and 6-month follow-up. A paired $t$-test was used to compare the change in mean MAU after treatment. Results. A total of 778 patients diagnosed with gout were screened for microalbuminuria. Among them, 114 (14.6\%) had urinary microalbumin levels of $>30.0 \mathrm{mg} / \mathrm{L}$ during presentation. Mean MAU level among those with microalbuminuria was $132.4 \pm 124.6 \mathrm{mg} / \mathrm{L}$. Thirty-five patients had concomitant HTN and were put on ARBs (20 mg of telmisartan). All received $40 \mathrm{mg}$ of febuxostat. In patients with ARBs, MAU reduced significantly after 3 months of treatment with ARBs. Reduction in MAU in those without ARBs was seen after the 6-month follow-up, and the change was statistically significant. Conclusions. There is significant reduction in MAU after the use of urate-lowering drugs in patients with gout.
\end{abstract}

\section{Introduction}

Gout is increasingly recognized as one of the major health problems in recent decades [1]. It is usually associated with an elevated serum uric acid (SUA) level. Higher SUA levels above the saturation threshold tend to form and deposit monosodium urate (MSU) crystals in and around joints [2]. Uric acid is a purine metabolic product excreted mainly by kidneys ( $2 / 3^{\text {rd }}$ amount) [3]. Apart from hyperuricemia, several risk factors for development of gout have been postulated in different studies including genetic factors, dietary factors, alcohol consumption, diuretic use, and renal disease [4]. There are studies showing the close association of hyperuricemia or gout with metabolic syndrome [5-7]. Gout can be considered as a risk factor for hypertension (HTN), cardiovascular disease, chronic kidney disease (CKD), and diabe- tes [8]. Few studies suggest that uric acid contributes to increased inflammation leading to CKD progression, thus demonstrating the relationship between serum urate levels and kidney function. While hyperuricemia can cause the progression of renal disease, kidney dysfunction itself can increase serum urate levels due to glomerular damage leading to reduced excretion of serum uric acid $[6,7]$.

Microalbuminuria (MAU) is defined as the persistent elevation of albumin in urine ranging from 30 to $300 \mathrm{mg}$ /day in a spot urine sample [9]. MAU is an established marker of risk calculation for the presence of cardiovascular disease and also predicts the progression of nephropathy when its level peaks above $300 \mathrm{mg} /$ day, reflecting generalized endothelial damage $[10,11]$. Allopurinol and febuxostat are the frequently used urate-lowering agents (ULT) which cause competitive inhibition of xanthine oxidase [12]. Although some 
of the studies have suggested association between these ULTs (febuxostat and allopurinol) and kidney function, only few studies have reported the effect of urate-lowering therapy in preventing deterioration of kidney function or reduction in microalbuminuria $[13,14]$. Change in the serum uric acid level and the urinary microalbumin level with treatment of gout is yet to be assessed clearly. This study was aimed at studying the prevalence of microalbuminuria in gout patients and the effect of urate-lowering therapy on urinary microalbumin levels.

\section{Materials and Methods}

2.1. Ethics Statement. This was a prospective, observational, cohort study conducted at the National Center for Rheumatic Diseases (NCRD), a tertiary-level rheumatology center in Nepal. Written informed consent from each patient was obtained, and ethical approval was taken from the institutional review board.

2.2. Study Population. Consecutive patients diagnosed with gout based on the 2015 ACR/EULAR criteria [15] attending the rheumatology clinic at the National Center for Rheumatic Diseases, Kathmandu, Nepal, from June 2016 to May 2019 were screened for microalbuminuria. Patients with severe cardiac, renal, neurological, and other inflammatory diseases and those who are receiving or have received urate-lowering therapy, angiotensin-converting enzyme inhibitor (ACE-I), or angiotensin receptor blocker (ARB) in the last 3 months were excluded. Pregnant and lactating mothers were also excluded from the study.

\subsection{Treatment Groups}

2.3.1. ARB Group. Patients with microalbuminuria and concomitant HTN were assigned to ARB (20 mg of telmisartan, once daily) and febuxostat.

2.3.2. Non-ARB Group. Those with microalbuminuria but no concomitant HTN were given febuxostat only. The dose of urate-lowering therapy (febuxostat) was initiated at $40 \mathrm{mg}$ once a day and was titrated to lower serum urate levels to 4 to $6 \mathrm{mg} / \mathrm{dL}$.

2.4. Collection of Data. Sociodemographic profile and clinical data were recorded in a predesigned Excel sheet at baseline. Serum uric acid level, spot urinary microalbumin level, blood sugar level, lipid profile, serum creatinine, and blood pressure were measured at baseline, 3-month follow-up, and 6-month follow-up. HTN was defined as systolic BP $\geq 140 \mathrm{mmHg}$ and/or diastolic BP $\geq 90 \mathrm{mmHg}$ and/or current use of antihypertensive medication [16]. Likewise, microalbuminuria was defined as a spot urinary albumin level between 30 and $300 \mathrm{mg} / \mathrm{L}$ [9].

2.5. Laboratory Methods. SUA was measured by the enzymatic uricase method using an automated analyzer (Erba XL 200; Erba Diagnostics, Mannheim, Germany). Urine microalbumin was measured by antigen antibody reaction, using an automated analyzer (Erba XL 200; Erba Diagnostics,
Mannheim, Germany). Blood pressure was measured using a sphygmomanometer.

2.6. Statistical Analysis. Statistical analysis was carried out with the help of SPSS software (version 16.0 system for Windows (SPSS, Chicago, IL)). A paired $t$-test was performed to compare the difference between pre- and post treatment mean serum uric acid levels and urinary microalbumin levels within the group. An independent $t$-test was done to compare mean serum uric acid levels and urinary microalbumin levels in between the two groups. All $p$ values presented were two-tailed, and a value of 0.05 or less was considered as statistically significant.

\section{Results}

Out of 778 patients with gout screened, $14.6 \%$ patients showed microalbuminuria during presentation. The mean age of 114 patients with microalbuminuria was $51.1 \pm 12.6$ years with mean BMI of $27.0 \pm 5.3 \mathrm{~kg} / \mathrm{m}^{2}$. The mean MAU level among those with microalbuminuria was $132.4 \pm$ $124.6 \mathrm{mg} / \mathrm{L}$. Among the 114 patients with high MAU, 35 patients were found to have concomitant HTN and were kept on telmisartan. Other clinic-demographic profiles of the two study groups (ARB and non-ARB) are shown in Table 1.

Serum UA levels decreased to the target level of 4$6 \mathrm{mg} / \mathrm{dL}$ in both groups post treatment. There was no significant difference in UA levels at the 3- and 6-month follow-ups in between the 2 treatment groups as shown by the independent $t$-test ( $p$ values 0.576 and 0.219 for the 3- and 6-month follow-ups, respectively). In patients on $\mathrm{ARB}$, the mean MAU levels at baseline, 3-month follow-up, and 6-month follow-up were $127.9 \pm 111.6,72.0 \pm 109.3$, and $44.9 \pm 61.6 \mathrm{mg} / \mathrm{L}$, respectively. The reduction in MAU in this group was statistically significantly after 3 months of treatment with ARBs (Table 2). In the patients without $\mathrm{ARB}$, the mean MAU levels at baseline, 3-month followup, and 6-month follow-up were $69.6 \pm 14.6 \mathrm{mg} / \mathrm{L}, 42.2 \pm$ $54.4 \mathrm{mg} / \mathrm{L}$, and $15.7 \pm 4.9 \mathrm{mg} / \mathrm{L}$, respectively. Significant reduction in MAU in those without $A R B$ was seen after the 6-month follow-up (Table 3).

\section{Discussion}

Gout is a common rheumatic disorder with a prevalence of $0.1-10 \%$ of the general population with higher prevalence in the male gender before the menopausal age of females [17]. It is characterized by hyperuricemia and deposition of urate crystals in the tissues, of which the most common is the synovium $[2,18,19]$. However, only $5 \%$ of patients with hyperuricemia develop gout [2]. It has been seen that the disorder, either gout or hyperuricemia, has been associated with derangement of various metabolic parameters like microalbuminuria, dyslipidemia, nonalcoholic steatohepatitis, chronic kidney disease, and cardiovascular diseases [20-24].

The relationship between uric acid and the urinary albumin excretion rate is a well-known fact. Increased prevalence of microalbuminuria with or without CKD in patients with gout or hyperuricemia has been seen [25]. In a study done 
TABLE 1: Baseline characteristics of the subjects $(n=114)$.

\begin{tabular}{|c|c|c|c|}
\hline & ARB group $(n=35)$ & Non-ARB group $(n=79)$ & $p$ value \\
\hline Age (years) mean $\pm S D$ & $51.4 \pm 12.4$ & $50.9 \pm 12.8$ & 0.847 \\
\hline Male/female $(n)$ & $33 / 2$ & $72 / 7$ & \\
\hline $\mathrm{BMI}\left(\mathrm{kg} / \mathrm{m}^{2}\right)$ mean $\pm \mathrm{SD}$ & $25.6 \pm 5.8$ & $27.5 \pm 4.9$ & 0.082 \\
\hline Serum urate $(\mathrm{mg} / \mathrm{dL})$ mean $\pm \mathrm{SD}$ & $6.9 \pm 2.4$ & $7.5 \pm 1.9$ & 0.183 \\
\hline $\mathrm{SBP}(\mathrm{mmHg})$ mean $\pm \mathrm{SD}$ & $135.5 \pm 23.6$ & $126.4 \pm 18.2$ & 0.324 \\
\hline $\mathrm{DBP}(\mathrm{mmHg})$ mean $\pm \mathrm{SD}$ & $89.1 \pm 14.2$ & $86.5 \pm 9.4$ & 0.035 \\
\hline $\mathrm{DM}(\%)$ & 9.1 & 15.2 & \\
\hline Thyroid disease (\%) & 12.1 & 3.8 & \\
\hline Hyperlipidemia $(n)$ & 54.5 & 59.5 & \\
\hline $\mathrm{MAU}(\mathrm{mg} / \mathrm{L})$ mean $\pm \mathrm{SD}$ & $127.9 \pm 111.6$ & $69.6 \pm 14.6$ & 0.056 \\
\hline CRP (mg/L) median & 5.0 & 8.0 & 0.696 \\
\hline Waist circumference $(\mathrm{cm})$ & $98.0 \pm 8.5$ & $99.4 \pm 9.7$ & 0.674 \\
\hline $\mathrm{TG}(\mathrm{mg} / \mathrm{dL})$ mean $\pm \mathrm{SD}$ & $286.6 \pm 278.8$ & $197.9 \pm 122.1$ & 0.011 \\
\hline $\mathrm{HDL}(\mathrm{mg} / \mathrm{dL})$ mean $\pm \mathrm{SD}$ & $42.7 \pm 10.0$ & $46.3 \pm 12.6$ & 0.140 \\
\hline $\mathrm{RBS}(\mathrm{mg} / \mathrm{dL})$ mean $\pm \mathrm{SD}$ & $116.6 \pm 44.8$ & $113.3 \pm 42.7$ & 0.747 \\
\hline Alcohol consumption $(n)$ & 8 & 22 & \\
\hline Smoking $(n)$ & 4 & 6 & \\
\hline
\end{tabular}

BMI: body mass index; SBP: systolic blood pressure; DBP: diastolic blood pressure; ARB: angiotensin receptor blocker; MAU: urinary microalbumin; RBS: random blood sugar; CRP: C-reactive protein; LDL: low-density lipoprotein; HDL: high-density lipoprotein; TCH: total cholesterol; TG: triglyceride.

TABLE 2: Change in MAU and SUA after 3 and 6 months of treatment in ARB group $(n=35)$.

\begin{tabular}{|c|c|c|c|c|c|}
\hline & Baseline & 3 months & $p$ value* & 6 months & $p$ value* \\
\hline Urinary microalbumin (MAU) & $127.9 \pm 111.6$ & $72.0 \pm 109.3$ & 0.02 & $44.9 \pm 61.6$ & 0.07 \\
\hline Serum uric acid (SUA) & $6.9 \pm 2.4$ & $5.8 \pm 2.43$ & 0.038 & $5.3 \pm 2.2$ & 0.348 \\
\hline
\end{tabular}

*Paired $t$-test.

TABLE 3: Change in MAU and SUA after 3 and 6 months of treatment in non-ARB group $(n=79)$.

\begin{tabular}{|c|c|c|c|c|c|}
\hline & Baseline & 3 months & $p$ value* & 6 months & $p$ value* \\
\hline Urinary microalbumin (MAU) & $69.6 \pm 14.6$ & $42.2 \pm 54.4$ & 0.14 & $15.7 \pm 4.9$ & 0.04 \\
\hline Serum uric acid (SUA) & $7.5 \pm 1.9$ & $6.2 \pm 2.2$ & 0.082 & $5.7 \pm 2.5$ & 0.70 \\
\hline
\end{tabular}

${ }^{*}$ Paired $t$-test.

in rats by Mazzali et al., it was shown that even mild hyperuricemia can cause renal injury and albuminuria [26]. Also, many authors have focused on the relationship between albuminuria and SUA documenting positive association between them in the general population and in patients with diabetes mellitus and hypertension [27-29]. It seems that as increased serum uric acid predisposes to nephropathy and loss of kidney function, lowering its levels can be helpful in preventing it. In a murine study, induced hyperuricemia resulted in HTN, renal arteriolopathy along with glomerular HTN with tubulointerstitial inflammation, and fibrosis. When hyperuricemia was corrected to normal levels with uratelowering agents, the above-mentioned manifestations were all corrected [26, 30-32].

Urate-lowering therapy leads to suppression of the renin-angiotensin-aldosterone axis, improves nitric oxide bioavailability as well as endothelial function, and reduces the oxidative stress and urinary inflammatory biomarkers [30, 33-35]. It has been reported that kidney function improved by treatment with allopurinol or febuxostat for gout $[13,36]$. Our observation suggests that treatment of gout with ULT can result in improvement of associated microalbuminuria. Similar observations were made in other studies. In a study by Ueno, kidney function was ameliorated and albuminuria was decreased in patients with gout on ULT suggesting that ULT could prevent kidney function if started as early as possible [37]. Pai et al. found an interesting observation that allopurinol was effective in reducing uric acid and preventing renal dysfunction, hypertension, proteinuria, renal hypertrophy, vascular disease, and renal scarring [38]. Another drug, benziodarone, a uricosuric agent, however, did not account for any of the above-mentioned benefits, and lowering of uric acid was less effective compared to allopurinol and febuxostat in a previous study strengthening the hypothesis [39]. 
In our study, we could observe that the serum uric acid level was not dramatically reduced in either group with the mean level remaining more than $5 \mathrm{mg} / \mathrm{dL}$, though the change reached the level of statistical significance compared with the baseline in one group. Thus, the improvement in microalbuminuria might be due to the class effect of febuxostat rather than the absolute effect of serum urate levels, as also suggested by the findings of the study on benziodarone [39]. Microalbuminuria thus showed an improvement with febuxostat in patients with gout. A statistically significant change was seen only after 6 months of treatment with febuxostat, whereas more rapid improvement at 3 months, as expected, was seen with concomitant use of ARB. However, it is not clear whether ULT is indicated for patients with conditions like cardiovascular disease with microalbuminuria but without CKD or gout.

\section{Conclusion}

Urate-lowering therapy reduces the urinary microalbumin levels in the patients with gout.

\section{Limitations}

The major limitation of this study was the study being conducted in a single center. Likewise, other urate-lowering agents were not used and compared with febuxostat.

\section{Data Availability}

The data used to support the findings of this study are available from the corresponding author upon request.

\section{Disclosure}

The abstract of this manuscript was accepted and presented as a poster at the Indian Rheumatology Association Conference (IRACON) 2019.

\section{Conflicts of Interest}

The authors declare that they have no conflicts of interest.

\section{Acknowledgments}

The authors would like to thank Ms. Rakhshya Joshi, research officer at NCRD, for her support in data collection. The authors also thank Dr. Jayanti Rai, rheumatologist at NCRD, for her help in patient care.

\section{References}

[1] S. J. Lee, J. D. Hirsch, R. Terkeltaub et al., "Perceptions of disease and health-related quality of life among patients with gout," Rheumatology (Oxford), vol. 48, no. 5, pp. 582-586, 2009.

[2] G. Ragab, M. Elshahaly, and T. Bardin, "Gout: an old disease in new perspective - a review," Journal of Advanced Research, vol. 8, no. 5, pp. 495-511, 2017.
[3] R. J. Johnson, D. H. Kang, D. Feig et al., "Is there a pathogenetic role for uric acid in hypertension and cardiovascular and renal disease?," Hypertension, vol. 41, no. 6, pp. 1183$1190,2003$.

[4] M. Doherty, "New insights into the epidemiology of gout," Rheumatology (Oxford), vol. 48, Supplement 2, pp. ii2-ii8, 2009.

[5] X. Sui, T. S. Church, R. A. Meriwether, F. Lobelo, and S. N. Blair, "Uric acid and the development of metabolic syndrome in women and men," Metabolism, vol. 57, no. 6, pp. 845-852, 2008.

[6] V. Bhole, J. W. Choi, S. W. Kim, M. de Vera, and H. Choi, "Serum uric acid levels and the risk of type 2 diabetes: a prospective study," The American Journal of Medicine, vol. 123, no. 10, pp. 957-961, 2010.

[7] A. F. Rubio-Guerra, H. Morales-López, A. Garro-Almendaro et al., "Circulating levels of uric acid and risk for metabolic syndrome," Current Diabetes Reviews, vol. 13, no. 1, pp. 8790, 2017.

[8] S. K. Kim, "Interrelationship of uric acid, gout, and metabolic syndrome: focus on hypertension, cardiovascular disease, and insulin resistance," Journal of Rheumatic Diseases, vol. 25, no. 1, pp. 19-27, 2018.

[9] J. M. Halimi, S. Hadjadj, V. Aboyans et al., "Microalbuminuria and urinary albumin excretion: French clinical practice guidelines," Diabetes \& Metabolism, vol. 33, no. 4, pp. 303-309, 2007.

[10] G. Bellomo, P. Berardi, P. Saronio et al., "Microalbuminuria and uric acid in healthy subjects," Journal of Nephrology, vol. 19, no. 4, pp. 458-464, 2006.

[11] M. F. Yuyun, A. I. Adler, and N. J. Wareham, "What is the evidence that microalbuminuria is a predictor of cardiovascular disease events?," Current Opinion in Nephrology and Hypertension, vol. 14, no. 3, pp. 271-276, 2005.

[12] E. Suresh and P. Das, "Recent advances in management of gout," QJM, vol. 105, no. 5, pp. 407-417, 2012.

[13] D. Sircar, S. Chatterjee, R. Waikhom et al., "Efficacy of Febuxostat for Slowing the GFR Decline in Patients With CKD and Asymptomatic Hyperuricemia: A 6-Month, Double-Blind, Randomized, Placebo- Controlled Trial," American Journal of Kidney Diseases, vol. 66, no. 6, pp. 945-950, 2015.

[14] P. Liu, Y. Chen, B. Wang, F. Zhang, D. Wang, and Y. Wang, "Allopurinol treatment improves renal function in patients with type 2 diabetes and asymptomatic hyperuricemia: 3year randomized parallel-controlled study," Clinical Endocrinology, vol. 83, no. 4, pp. 475-482, 2015.

[15] T. Neogi, T. L. Jansen, N. Dalbeth et al., "2015 gout classification criteria: an American College of Rheumatology/European League Against Rheumatism collaborative initiative," Arthritis \& Rhematology, vol. 67, no. 10, pp. 2557-2568, 2015.

[16] G. M. Gabb, A. A. Mangoni, C. S. Anderson et al., "Guideline for the diagnosis and management of hypertension in adults 2016," The Medical Journal of Australia, vol. 205, no. 2, pp. 85$89,2016$.

[17] C. F. Kuo, M. J. Grainge, W. Zhang, and M. Doherty, "Global epidemiology of gout: prevalence, incidence and risk factors," Nature Reviews Rheumatology, vol. 11, no. 11, pp. 649-662, 2015.

[18] D. J. McCarty Jr., P. Phelps, and J. Pyenson, "Crystal-induced inflammation in canine joints. I. An experimental model with quantification of the host response," The Journal of Experimental Medicine, vol. 124, no. 1, pp. 99-114, 1966. 
[19] E. W. Campion, R. J. Glynn, and L. O. DeLabry, “Asymptomatic hyperuricemia. Risks and consequences in the Normative Aging Study," The American Journal of Medicine, vol. 82, no. 3, pp. 421-426, 1987.

[20] H. Latif, A. Iqbal, R. Rathore, and N. F. Butt, "Correlation between serum uric acid level and microalbuminuria in type2 diabetic nephropathy," Pakistan Journal of Medical Sciences, vol. 33, no. 6, pp. 1371-1375, 2017.

[21] T. C. Peng, C. C. Wang, T. W. Kao et al., "Relationship between hyperuricemia and lipid profiles in US adults," BioMed Research International, vol. 2015, Article ID 127596, 7 pages, 2015.

[22] E. Sertoglu, C. N. Ercin, G. Celebi et al., "The relationship of serum uric acid with non-alcoholic fatty liver disease," Clinical Biochemistry, vol. 47, no. 6, pp. 383-388, 2014.

[23] R. J. Johnson, T. Nakagawa, D. Jalal, L. G. Sanchez-Lozada, D. H. Kang, and E. Ritz, "Uric acid and chronic kidney disease: which is chasing which?," Nephrology, Dialysis, Transplantation, vol. 28, no. 9, pp. 2221-2228, 2013.

[24] M. L. Muiesan, C. Agabiti-Rosei, A. Paini, and M. Salvetti, "Uric acid and cardiovascular disease: an update," European Cardiology Review, vol. 11, no. 1, pp. 54-59, 2016.

[25] H. Y. Chang, P. H. Lee, C. C. Lei et al., "Hyperuricemia is an independent risk factor for new onset micro-albuminuria in a middle-aged and elderly population: a prospective cohort study in Taiwan," PLoS One, vol. 8, no. 4, article e61450, 2013.

[26] M. Mazzali, J. Kanellis, L. Han et al., "Hyperuricemia induces a primary renal arteriolopathy in rats by a blood pressureindependent mechanism," American Journal of Physiology. Renal Physiology, vol. 282, no. 6, pp. F991-F997, 2002.

[27] X. H. Fan, J. F. Cai, B. X. Gao et al., "The relationship between urinary albumin excretion and serum uric acid in general population," Zhonghua Nei Ke Za Zhi, vol. 50, no. 7, pp. 550-554, 2011.

[28] Y. Hayashino, S. Okamura, S. Tsujii, and H. Ishii, “Association of serum uric acid levels with the risk of development or progression of albuminuria among Japanese patients with type 2 diabetes: a prospective cohort study [Diabetes Distress and Care Registry at Tenri (DDCRT 10)]," Acta Diabetologica, vol. 53, no. 4, pp. 599-607, 2016.

[29] J. P. Forman, L. Scheven, P. E. de Jong, S. J. Bakker, G. C. Curhan, and R. T. Gansevoort, "Association between sodium intake and change in uric acid, urine albumin excretion, and the risk of developing hypertension," Circulation, vol. 125, no. 25, pp. 3108-3116, 2012.

[30] M. Mazzali, J. Hughes, Y. G. Kim et al., "Elevated uric acid increases blood pressure in the rat by a novel crystalindependent mechanism," Hypertension, vol. 38, no. 5, pp. 1101-1106, 2001.

[31] L. G. Sánchez-Lozada, E. Tapia, C. Avila-Casado et al., "Mild hyperuricemia induces glomerular hypertension in normal rats," American Journal of Physiology-Renal Physiology, vol. 283, no. 5, pp. F1105-F1110, 2002.

[32] L. G. Sanchez-Lozada, E. Tapia, V. Soto et al., "Treatment with the xanthine oxidase inhibitor febuxostat lowers uric acid and alleviates systemic and glomerular hypertension in experimental hyperuricaemia," Nephrology, Dialysis, Transplantation, vol. 23, no. 4, pp. 1179-1185, 2008.

[33] K. M. Talaat and A. R. El-Sheikh, "The effect of mild hyperuricemia on urinary transforming growth factor beta and the pro- gression of chronic kidney disease," American Journal of Nephrology, vol. 27, no. 5, pp. 435-440, 2007.

[34] R. Butler, A. D. Morris, J. J. Belch, A. Hill, and A. D. Struthers, "Allopurinol normalizes endothelial dysfunction in type 2 diabetics with mild hypertension," Hypertension, vol. 35, no. 3, pp. 746-751, 2000.

[35] Y. P. Siu, K. T. Leung, M. K. Tong, and T. H. Kwan, "Use of allopurinol in slowing the progression of renal disease through its ability to lower serum uric acid level," American Journal of Kidney Diseases, vol. 47, no. 1, pp. 51-59, 2006.

[36] H. Ito, S. Antoku, M. Abe et al., "Comparison of the renoprotective effect of febuxostat for the treatment of hyperuricemia between patients with and without type 2 diabetes mellitus: a retrospective observational study," Internal Medicine, vol. 55, no. 22, pp. 3247-3256, 2016.

[37] N. Ueno, "Urate-lowering therapy ameliorates kidney function in type 2 diabetes patients with hyperuricemia," Journal of Clinical Medical Research, vol. 9, no. 12, pp. 1007-1012, 2017.

[38] B. H. Pai, G. Swarnalatha, R. Ram, and K. V. Dakshinamurty, "Allopurinol for prevention of progression of kidney disease with hyperuricemia," Indian Journal of Nephrology, vol. 23, no. 4, pp. 280-286, 2013.

[39] D. H. Kang, T. Nakagawa, L. Feng et al., "A role for uric acid in the progression of renal disease," Journal of the American Society of Nephrology, vol. 13, no. 12, pp. 2888-2897, 2002. 